\title{
Glucose effects on complex memory and nonmemory tasks: The influence of age, sex, and glucoregulatory response
}

\author{
SUZANNE CRAFT, CHRISTOPHER MURPHY, and JENNIFER WEMSTROM \\ Washington University, St. Louis, Missouri
}

\begin{abstract}
Complex tests of declarative memory, working memory, procedural learning, and response generation and inhibition were administered to 27 younger adults $(M=20.8$ years $)$ and 32 older adults ( $M=68.5$ years) following ingestion of saccharin $(23.7 \mathrm{mg})$ or glucose $(50 \mathrm{~g})$. The effects of glucose administration were examined along three dimensions: age, sex, and glucoregulatory response, defined as the degree to which blood glucose levels returned to baseline following glucose administration. Glucose administration significantly enhanced performance on the declarative memory measure paragraph recall for older males and younger males whose blood glucose returned to similar levels. Younger men whose blood glucose levels were lowest $60 \mathrm{~min}$ after glucose ingestion showed memory deterioration. Glucose administration did not affect paragraph recall for the other groups, nor were effects noted for any group for most other cognitive measures. These results suggest that the effects of glucose administration are primarily restricted to declarative memory independent of task complexity, and that age, sex, and glucoregulatory response may influence hyperglycemic memory enhancement.
\end{abstract}

The brain uses glucose as its primary substrate for cognitive activity (Raichle, Herscovitch, Mintun, Martin, \& Powers, 1984). It is not surprising, therefore, that conditions that affect glucose regulation and utilization may also affect cognitive function. One such condition is insulin-dependent diabetes mellitus. Cognitive deficits, particularly in memory abilities, have been documented in diabetic patients who have early age of onset and severe hypoglycemic episodes (Holmes, 1990; Ryan, in press).

A growing body of evidence suggests that normal aging may constitute another condition in which greater vulnerability to disrupted glucose regulation can contribute to memory impairment. Gold and colleagues have demonstrated that by increasing plasma glucose levels and thereby enhancing the availability of glucose to the brain, memory performance is improved in aged rodents and humans (Gold \& Stone, 1988; GonderFrederick et al., 1987; Manning, Jones, Hall, \& Gold, 1990; Manning, Parsons, \& Gold, 1992). General findings from these studies indicate that (1) memory tests are selectively affected, and (2) younger adults show less memory enhancement following glucose administration than do older adults with good glucose regulation (Azari, 1991; Hall, Gonder-Frederick, Chewing, Silveira, \& Gold, 1989).

This study was supported by a grant from the National Institute on Aging (NIA AG 10880). C.M. was supported by NIA Grant AG0030. Address correspondence to S. Craft, GRECC-182B, Seattle/American Lake Veterans Affairs Medical Center, Tacoma, WA 98493.
Older adults' greater sensitivity to hyperglycemic effects presumably reflects the disruption of glucose regulation or utilization that becomes more common with aging. Indeed, evidence to support this possibility is provided by estimates of the widespread prevalence of adult-onset diabetes among the elderly. Other authors have noted that the mild decline in memory function observed with aging is more pronounced in adults with poor glucose regulation, suggesting a link between agerelated changes in glucose regulation and memory performance (Gold \& Stone, 1988; Parsons \& Gold, 1992).

The question of whether hyperglycemic effects are specific to memory ability has important implications for the mechanisms that produce hyperglycemic facilitation. Candidate mechanisms include enhanced neurotransmitter synthesis through glycolytic production of acetyl-CoA, a substrate for acetylcholine, or reduced opiate turnover and related inhibition in the hippocampus (Gold, Vogt, \& Hall, 1986). An additional mechanism has been suggested by a study of normal elderly adults and patients with Alzheimer's disease, in which hyperglycemia-induced insulin elevations were related to changes in memory performance (Craft et al., 1993). Insulin receptors in the brain are densely represented in the hippocampus, hypothalamus, and olfactory bulb (Unger et al., 1989). Moderate doses of insulin have also been shown to increase firing of hippocampal neurons (Palovcik, Phillips, Kappy, \& Raizada, 1984). Thus, elevated insulin in response to hyperglycemia may promote glucose utilization in the hippocampus and subsequently improve memory ability. 
All three mechanisms presumably produce enhancement of hippocampal function, thereby accounting for the specificity of glucose effects to memory. It is possible, however, that previous studies have not assessed an adequate range of cognitive abilities in order to definitively determine such specificity. In general, nonmemory tests included in previous studies have not involved prolonged on-line processing of information or required complex manipulation of stimuli-abilities that have been categorized under the domain of working memory. They have also generally failed to include tests that have been shown to be mediated by specific neural systems other than the hippocampus in order to rule out generalized effects of hyperglycemia on brain metabolism. For example, studies have not examined tests of procedural learning, a form of nondeclarative memory thought to be mediated by the cerebellum or the basal ganglia (Squire, 1992), and tests of response generation or inhibition, generally thought to be mediated by the frontal lobes (Perret, 1974).

The specificity of hyperglycemic effects to older subjects can also be examined from this perspective. It is possible that previous studies have failed to include tests of sufficient sensitivity to detect hyperglycemic effects in younger subjects. Finally, no study to date has reported sex differences in hyperglycemic responsivity, despite strong evidence that such differences exist in basic aspects of glucose regulation and utilization (Armoni, Rafaeloff, Barzilai, Eitan, \& Karnieli, 1987; Hale, Wright, \& Nattrass, 1985; Paula et al., 1990).

In the present study we examined the effects of glucose administration on complex memory and nonmemory functions in younger and older men and women.

\section{METHOD}

\section{Subjects}

The subjects were 27 younger adults, aged $19-28$ years $(M=$ $20.8)$, and 32 older adults, aged $58-77$ years $(M=68.5)$. The subjects were recruited from volunteer pools maintained by the Washington University Department of Psychology and Aging and De- velopment Program. All the subjects received a screening questionnaire to determine that they were nondiabetic and in good health. Three of the older women were receiving estrogen replacement therapy. Demographic information for all the subject groups is presented in Table 1.

\section{Procedure}

The subjects participated in two morning sessions, following an overnight fast. After consent was obtained, a baseline fasting blood glucose level was measured using a One Touch II glucometer. Subjects with fasting glucose levels above $120 \mathrm{mg} / \mathrm{dl}$ were excluded from the study at that point. The subjects then consumed a 16-oz., orange-flavored beverage sweetened with either $50 \mathrm{~g}$ of dextrose or $2 \mathrm{ml}$ of "Sweet-10" sodium saccharin sweetener. Several drops of the saccharin solution were added to the dextrose preparation to equate flavor. The subjects were given $5 \mathrm{~min}$ in which to drink the beverage. The order of glycemic conditions was counterbalanced across subjects, and they were blind to condition. This procedure has been used in several other investigations of glucose effects on memory (Craft, Zallen, \& Baker, 1992; GonderFrederick et al., 1987; Hall et al., 1989; Manning et al., 1990).

In order to monitor the subjects' blood glucose levels, blood samples were taken using fingertip pin pricks prior to ingesting the beverage and $15,30,45$, and $60 \mathrm{~min}$ after ingestion of the beverage. Cognitive testing began 15 min after ingestion of the study beverage, and it was timed to coordinate with the blood samples so that each subject had comparable intervals between tasks and glucose measures. The protocol consisted of declarative, procedural, and working-memory measures, as well as tests of verbal fluency and response inhibition. Two comparable versions of the cognitive protocol were constructed and counterbalanced across glycemic conditions.

\section{Declarative Memory Measures}

Paragraph recall. This task has been used extensively in previous investigations into the effects of glucose on memory (Craft et al., 1993; Craft et al., 1992; Gonder-Frederick et al., 1987; Hall et al., 1989; Manning et al., 1990). The subjects listened to two brief narratives containing 25 information bits, and were asked to recall as much as they could remember immediately and $10 \mathrm{~min}$ after presentation. The subjects' responses were tape-recorded and scored by two blind raters. The subjects received 1 point for each bit recalled verbatim. Scores assigned by the two raters were averaged for each subject. Interrater reliability was .92 .

Modified California Verbal Learning Test. This task (Delis, Kramer, Kaplan, \& Ober, 1987; Delis et al., 1991) was included to test verbal learning over several trials. A modified presentation

Table 1

Mean Age, Body Mass Index $\left(\mathrm{kg} / \mathrm{m}^{2}\right)$, and Recovery Values (Blood Glucose at $60 \mathrm{~min}$ - Blood Glucose at Baseline in the Glucose Condition), With Standard Deviations for all Subject Groups

\begin{tabular}{|c|c|c|c|c|c|c|c|c|c|c|c|c|c|c|c|}
\hline \multicolumn{8}{|c|}{ Younger Adults } & \multicolumn{8}{|c|}{ Older Adults } \\
\hline \multicolumn{4}{|c|}{ Good Recovery } & \multicolumn{4}{|c|}{ Poor Recovery } & \multicolumn{4}{|c|}{ Good Recovery } & \multicolumn{4}{|c|}{ Poor Recovery } \\
\hline \multicolumn{2}{|c|}{ Male $(n=8)$} & \multicolumn{2}{|c|}{ Female $(n=6)$} & \multicolumn{2}{|c|}{ Male $(n=6)$} & \multicolumn{2}{|c|}{ Female $(n=7)$} & \multicolumn{2}{|c|}{ Male $(n=8)$} & \multicolumn{2}{|c|}{ Female $(n=8)$} & \multicolumn{2}{|c|}{ Male $(n=8)$} & \multicolumn{2}{|c|}{ Female $(n=8)$} \\
\hline$M$ & $S D$ & $M$ & $S D$ & $M$ & $S D$ & $M$ & $S D$ & $M$ & $S D$ & $M$ & $S D$ & $M$ & $S D$ & $M$ & $S D$ \\
\hline \multicolumn{16}{|c|}{ Age } \\
\hline 20.6 & 1.8 & 21.7 & 2.2 & 21.6 & 3.3 & 19.3 & 1.0 & 68.5 & 2.3 & 66.5 & 6.3 & 71.8 & 4.6 & 69.0 & 4.6 \\
\hline \multicolumn{16}{|c|}{ Body Mass Index* } \\
\hline 23.9 & 2.3 & 20.2 & 1.3 & 23.4 & 1.3 & 21.8 & 2.3 & 26.7 & 2.4 & 24.7 & 4.5 & 26.2 & 1.8 & 24.6 & 4.2 \\
\hline \multicolumn{16}{|c|}{ Recovery Index $\dagger$} \\
\hline 18.7 & 18.7 & 33.2 & 3.9 & 66.0 & 34.4 & 59.1 & 9.3 & 38.6 & 17.3 & 42.1 & 14.2 & 97.5 & 18.3 & 89.4 & 20.4 \\
\hline
\end{tabular}

*Older adults had significantly greater body mass than younger adults', and men had greater body mass than women's. fOlder adults had larger recovery values than younger adults'. 
was used for two alternate forms of the California Verbal Learning Test. In this task, the subjects listened to a list of 16 items drawn from four semantic categories and were asked to recall as many items as possible, in any order. This procedure was repeated two more times. The subjects then heard a second list and were asked to recall it. Immediately after this interference trial, they were asked to recall items from the first list. Free recall and recognition were then measured after a 15-min delay. Number correct was recorded for the first three trials, for short-delay recall, for long-delay recall, and for the recognition trial.

Pattern recall and recognition. The subjects viewed 3 checkerboard patterns presented on a single sheet of paper. Each pattern consisted of four randomly blackened squares on a $3 \times 3$ grid. They were told to study the 3 patterns for $10 \mathrm{sec}$. The stimuli were then removed, and the subjects were asked to reproduce each pattern on a sheet containing three blank grids. This process was repeated two more times. Free recall for the patterns was tested after a 10-min delay. Following the free recall, the subjects were asked to pick the 3 test patterns from an array of 12 checkerboard patterns that included 9 distractor patterns. Three scores were constructed: number correct for the first three trials, for delayed recall, and for the recognition trial.

\section{Procedural Memory Measure}

A serial reaction time task was used to measure implicit motor memory. As described by Nissen, Willingham, and Hartman (1989), the subjects sat in front of a computer screen with the middle and index fingers of each hand placed comfortably on four evenly spaced keys corresponding to four evenly spaced locations across the bottom of the computer screen. Their task was to press the key that corresponded to the location of an asterisk that appeared on the screen. Once a key was pressed, the asterisk appeared in another location, and the subjects pressed the key corresponding to the new location, and so on. There were five blocks of 100 trials each, with an initial block of 25 practice trials. For trials in the practice block and Blocks 1 and 5 , asterisk positions were randomly generated by the computer. Trials in Blocks 2, 3 , and 4 followed a repeating sequence of 10 positions. The 10position sequence was thus repeated 30 times across three blocks. Mean reaction times, measured as the time from the onset of the asterisk to the keypress, were averaged for each block for each subject. Normal subjects are generally unaware that there is a pattern in the trials, but nevertheless their reaction times decrease across the three patterned-sequence blocks. This decrease demonstrates motor learning, and it occurs without declarative, explicit knowledge of the pattern.

\section{Working-Memory Measure}

The Paced Serial Addition Test (Gronwall \& Sampson, 1974) was used as a measure of working memory. This task requires the subject to add 60 pairs of randomized digits, presented in a continuous string, at 2-sec intervals. Each digit is added to the digit immediately preceding it. For example, if the numbers presented were 1-3-5-4-6, the correct responses beginning at the digit 3 would be $4,8,9$, and 10 . The number of correct responses was recorded.

\section{Verbal Fluency Measure}

Word-list generation was included as a measure of the effects of hyperglycemia on a verbal nonmemory task that is sensitive to frontal lobe dysfunction (Benton, 1968). The subjects were given a different letter on each of three trials and were asked to generate, in $60 \mathrm{sec}$, as many words as possible that began with that letter. They were instructed not to include proper nouns, numbers, or different forms of the same word. Number of correct responses was summed across the three trials. The two alternate forms of this test were matched for total possible words and word frequency (Benton \& Hamsher, 1978).

\section{Response Inhibition Measure}

Stroop Color-Word Interference Test. This task (Stroop, 1935 ) is sensitive to frontal lobe lesions and has been shown to require effortful, on-line processing (Perret, 1974). The task included three conditions. In each condition, total reading time and errors were recorded. The first condition (word reading) required the subject to read 100 color words (red, green, blue), presented in rows on a sheet of paper, as quickly as possible. The second condition (color naming) required the subjects to name the colors of 100 colored blocks that were presented in rows on a sheet of paper. In the third condition (color-word interference), stimuli consisted of color names that were printed in discordant colors (e.g., the word blue printed in green letters). The subjects were asked to name the ink color of the printed words, and were thus required to inhibit the reading of the words themselves.

\section{RESULTS}

A repeated measures analysis of variance (ANOVA) was used to examine changes in cognitive performance and glucose levels following glucose administration in the younger and older male and female subjects. Previous studies have demonstrated that the degree to which blood glucose levels return to baseline following glucose administration is related to glucose effects on cognitive performance (Craft et al., 1992). Thus, an index of recovery was constructed by subtracting the baseline level from the final glucose level in the condition in which glucose was administered (final glucose - baseline glucose). With this index, large values reflect poorer recovery, and values approaching zero reflect better recovery. A median split was performed for each age group using the recovery index, with half of each age group thus designated as good or poor recovery. Mean recovery scores for each group are included in Table 1. For each cognitive score, a mixed, repeated measures ANOVA was conducted with the between-subject factors of age group (younger or older), recovery (good or poor), sex (male or female), and glycemic order (glucose or saccharin first), and the within-subjects factor of glycemic condition (glucose or saccharin). No significant effects were observed for order for any cognitive measure, and thus this factor was omitted from further analysis. In order to control for experimentwise Type I error, only those effects with $p<.01$ were considered statistically significant.

\section{Declarative Memory Measures}

For immediate and delayed paragraph-recall scores and the short- and long-delay recall scores from the California Verbal Learning Test, an additional withinsubjects factor of delay (immediate or delayed recall) was added to the glycemic condition, with age group, recovery, and sex remaining as between-subject factors.

For declarative memory measures, the effects of glucose administration were most clearly seen for paragraph recall. Interestingly, these effects differed for good- and poor-recovery male and female groups. The differences were reflected in a significant interaction between group, recovery, sex, and glycemic condition 
$[F(1,51)=7.50, p<.01]$. Post hoc analyses showed that the older men with good recovery performed significantly better in the glucose condition than in the saccharin condition $[F(1,7)=13.36, p<.01]$. This pattern was observed for immediate recall in each of the 8 subjects in this group, and for delayed recall in 7 of 8 subjects. The older men with poor recovery and the older women with good or poor recovery showed no significant differences between glycemic conditions.

Post hoc analysis revealed an interesting response pattern for the younger subjects. The younger men who were designated as having poor recovery showed significantly improved scores across both immediate and delayed recall in the glucose condition relative to the saccharin condition $[F(1,5)=14.78, p<.01]$, whereas the performance of the younger men with good recovery deteriorated with glucose administration $[F(1,7)=35.57$, $p<.001]$. Five of 6 younger men with poor recovery showed better immediate recall after glucose administration, and 4 of 6 showed improved delayed recall. In contrast, all 8 younger men designated as having good recovery showed decreased immediate recall, and 7 of 8 showed decreased delayed recall after glucose administration. The younger women with good or poor recovery did not show any significant differences in recall between glucose and saccharin conditions.

As expected, the main effects for group and withinsubjects effects for delay were observed for saccharin and glucose conditions (all $p \mathrm{~s}<.0001$ ), reflecting the poorer performance of the older group and lower delayed- than immediate-recall scores for all the groups. It should also be noted that no significant effects were observed for sex in the saccharin condition, suggesting that both the men and women had comparable memory performance at baseline. Data for paragraph recall are presented in Figures 1 and 2.

For all measures from the California Verbal Learning Test, main effects were again observed for group (all $p \mathrm{~s}<.0001$ ), reflecting better performance of the younger subjects. No interactions were noted for total immediate recall over the three presentations of the list or for short- and long-delay recall.

For the pattern-recall and recognition task, ceiling effects for the delayed-recall and recognition conditions precluded meaningful analysis of these data. For the total number of correct responses over the initial three trials, significant main effects for age group, reflecting higher scores for the younger subjects, were obtained in both glucose and saccharin conditions $(p s<.001)$. In addition, in the saccharin condition, an interaction was observed among age group, recovery, and sex $[F(1,45)=$ $8.44, p<.01]$. For the younger men and women and the older women, the poor- and good-recovery groups performed similarly. Surprisingly, however, the older men with poor recovery had better scores than the older men with good recovery $[F(1,14)=8.98, p<.01]$. No other interactions were noted.

In summary, for declarative memory measures, the effects of glucose administration were seen most clearly with paragraph recall-a measure that has been shown to be sensitive to glucose manipulation in several previous studies (Craft et al., 1993; Craft et al., 1992; Gonder-Frederick et al., 1987; Hall et al., 1989; Manning et al., 1990). Glucose effects were manifested primarily by the men; the older men with good recovery and the younger men with poor recovery showed memory facilitation with glucose administration, whereas the younger men with good recovery showed deterioration. This pattern of results was strongly reflected by individual subject data, suggesting that the observed effects were not due to a few outlying scores.

\section{Procedural Memory Measure}

For the serial reaction time task, a repeated measures ANOVA was conducted with block (1-5; Blocks 1 and 5 were random sequence, and Blocks 2,3 , and 4 were patterned sequence) and glycemic condition as the within-subjects factors, and group, recovery, and sex as the between-subject factors. Following the obtainment of significant effects or interactions involving the block factor, planned contrasts were conducted. Mean reaction times for patterned sequences in Block 4 were compared with means for random sequences in Block 5 to measure the amount of learning that had occurred in Block 4, and random sequence means from Block 1 were compared with random sequence means from Block 5 to determine any possible nonspecific effects of fatigue or task familiarity.

Glucose administration did not affect performance on this measure for any subject group. Main effects for age group were found for all conditions (all $p \mathrm{~s}<.0001$ ), reflecting the slower reaction times of the older group. A significant within-subjects effect was also observed for block $[F(4,176)=64.18, p<.0001]$. Planned contrasts revealed that reaction times for Block 4 were significantly faster than for Block $5[F(1,44)=156.61, p<$ $.0001]$. No significant differences were found between Blocks 1 and 5. No other significant effects or interactions were observed.

\section{Working-Memory Measure}

For the Paced Serial Addition Test, main effects for age group were found for both glycemic conditions (both $p s<.0001$ ), reflecting fewer correct responses for the older group compared with the younger group. Performance was unaffected by glucose administration.

\section{Verbal Fluency Measure}

No main effects or interactions were observed for word-list generation. The older subjects performed similarly to the younger subjects, and performance was unaffected by glucose administration.

\section{Response Inhibition Measure}

For the Stroop Color-Word Interference Test, repeated measures ANOVAs were conducted for time and error scores with task condition (word reading, color naming, or color-word interference) and glycemic condition as 


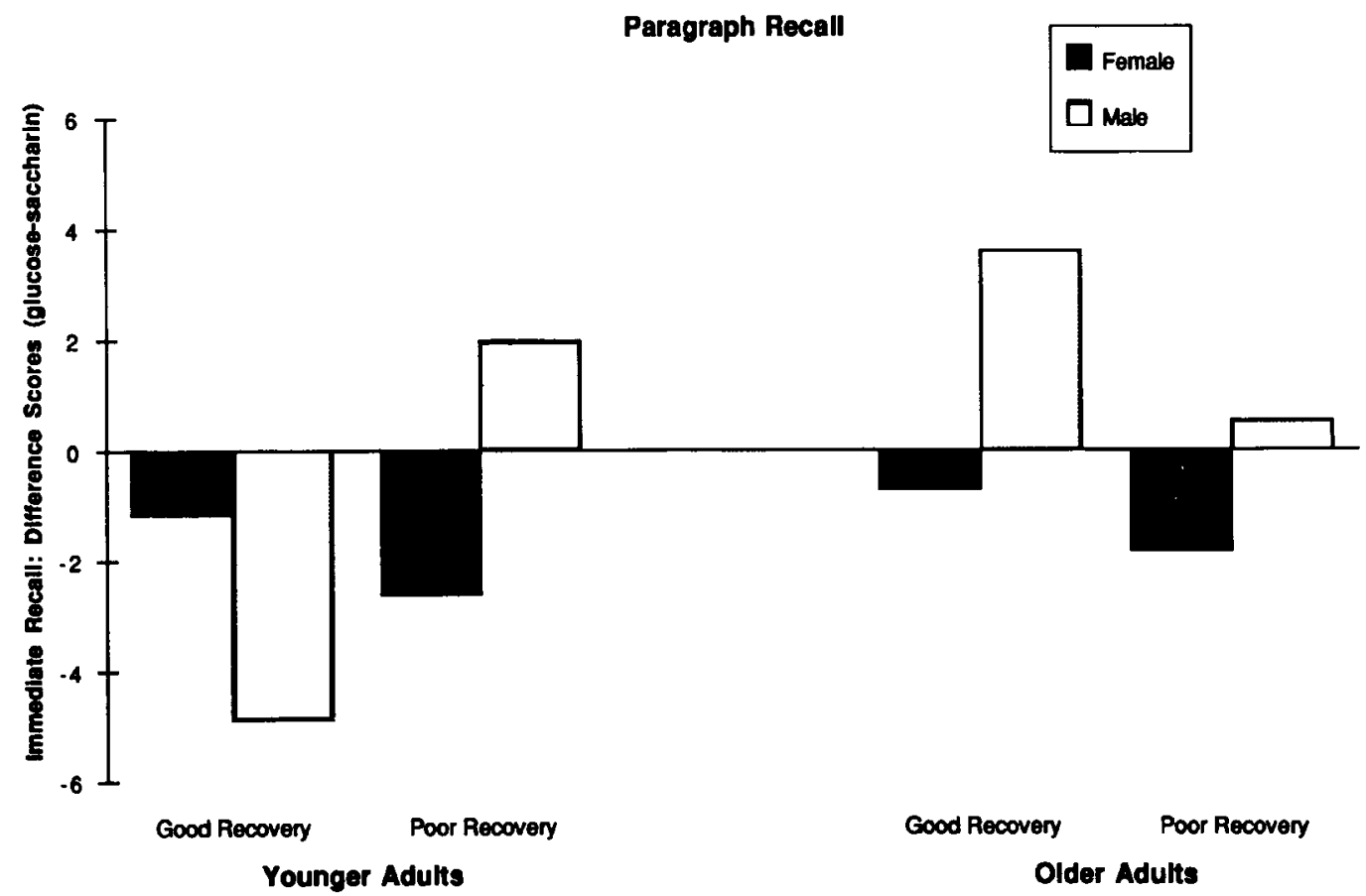

Figure 1. Mean immediate paragraph-recall difference scores (glucose-saccharin) for younger and older men and women with good and poor recovery.

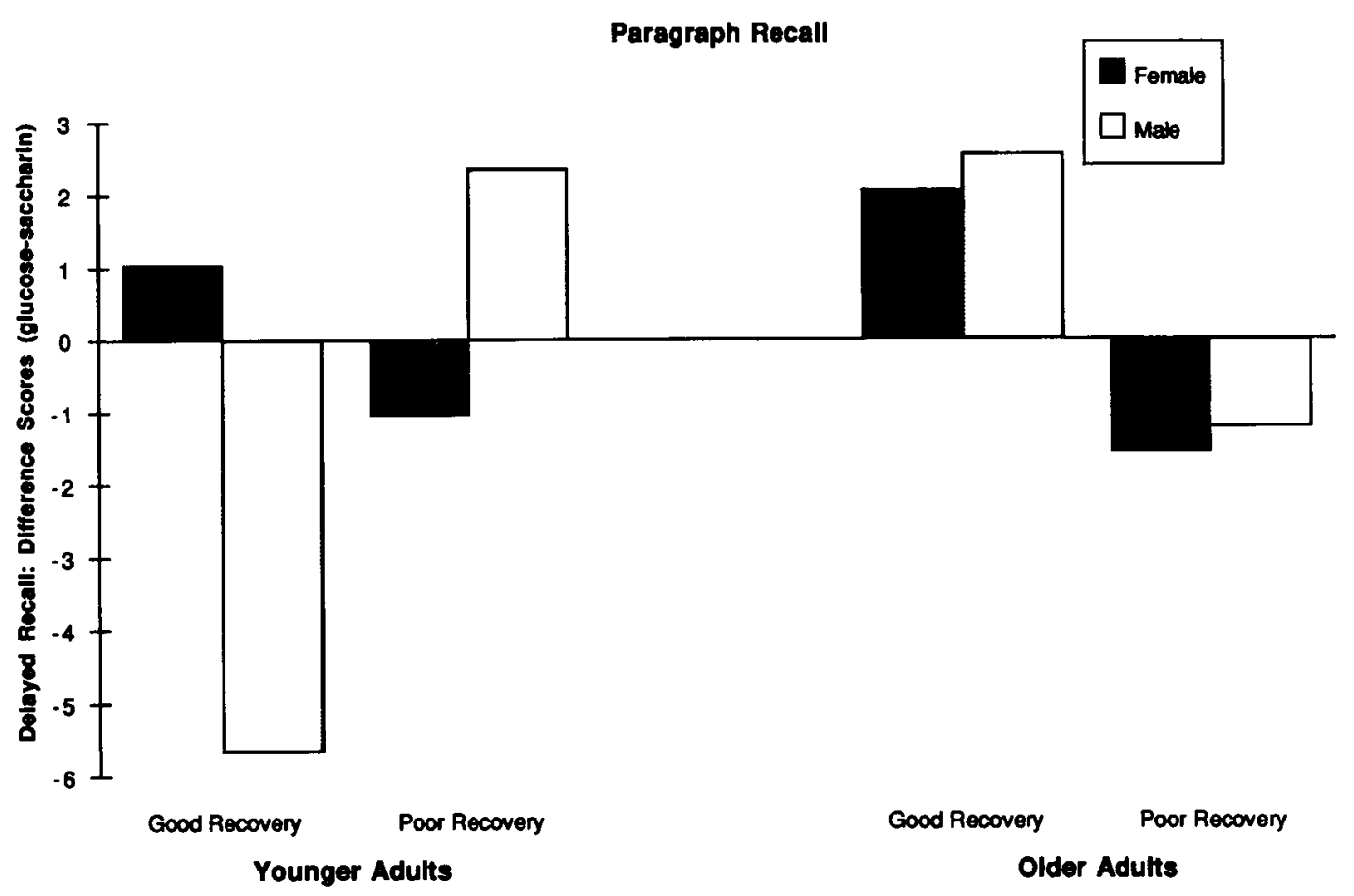

Figure 2. Mean delayed paragraph-recall difference scores (glucose-saccharin) for younger and older men and women with good and poor recovery. 


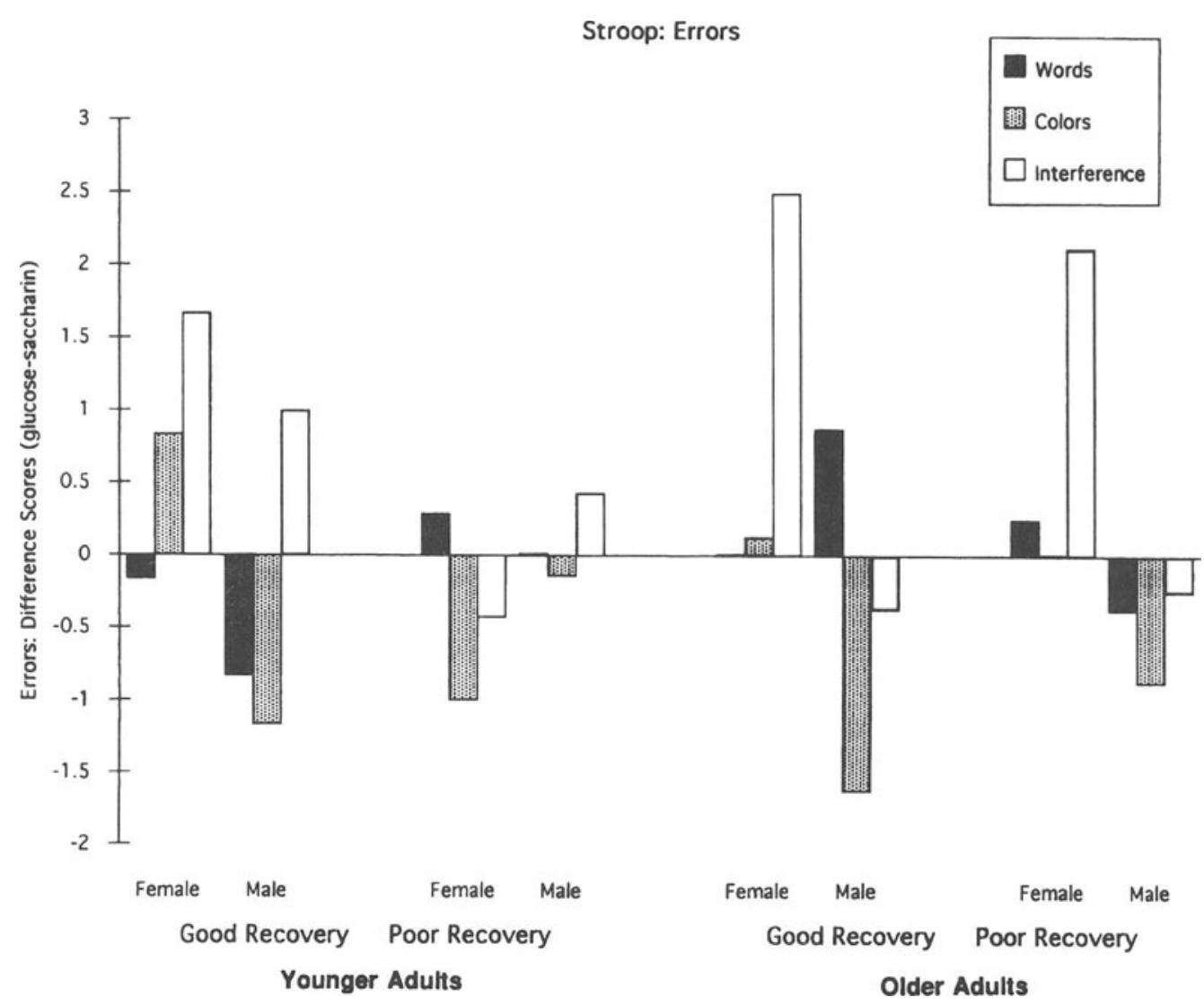

Figure 3. Mean Stroop error difference scores (glucose-saccharin) for younger and older men and women with good and poor recovery.

within-subjects factors. Following the obtainment of significant effects or interactions involving task condition, planned contrasts were conducted. Mean scores for the color-word interference condition were compared with scores for the reading and color-naming conditions to determine whether glucose administration affected the relative difficulty of the interference condition.

In general, similar glycemic effects were noted for all the subject groups for the Stroop test, consisting of faster response times and more errors in the interference condition following glucose administration. For time scores, a significant interaction was observed between the glycemic and task conditions $[F(2,94)=5.93, p<$ .01]. Planned comparisons revealed that all the subjects showed less of an increase in time scores from the wordreading condition to the interference condition after glucose administration $[F(1,47)=6.57, p<.01]$, with a similar trend noted between the color-naming and interference conditions $(p<.02)$.

The interaction between task condition and age group was also significant $[F(2,94)=41.08, p<.0001]$. Planned comparisons revealed that the increase in time from the word-reading to the interference condition was significantly greater for the older subjects than for the younger subjects $[F(1,47)=46.78, p<.0001]$, as was the increase from the color-naming to the interference condition $[F(1,47)=39.95, p<.0001]$. Finally, main effects for group and within-subjects effects for condition were noted (all $p s<.01$ ), reflecting the slower times of the older subjects in all conditions and slower times for all the subjects in the interference condition relative to the word-reading and color-naming conditions.

For error scores, a significant glycemic condition $\times$ task condition interaction was also observed $[F(2,100)=$ $5.31, p<.01]$. However, unlike the pattern of results observed for time scores, planned comparisons revealed poorer performance (more errors) in the interference condition relative to the color-naming condition after glucose administration $[F(1,50)=7.88, p<.01$; Figure 3$]$. There was only one significant main effect for sex in the colornaming/saccharin condition, reflecting more errors for male subjects $[F(1,50)=10.07, p<.01]$. As with time scores, a large effect was obtained for task condition $[F(2,100)=56.33, p<.0001]$. Planned comparisons revealed more errors in the interference condition compared with the word-reading and color-naming conditions $[F(1,50)=93.09$ and 24.40 , respectively, $p s<.0001]$.

\section{Glucose Levels}

A repeated measures ANOVA was conducted with blood-sample time (baseline and 15, 30, 45, and $60 \mathrm{~min}$ following beverage ingestion) and glycemic condition as 


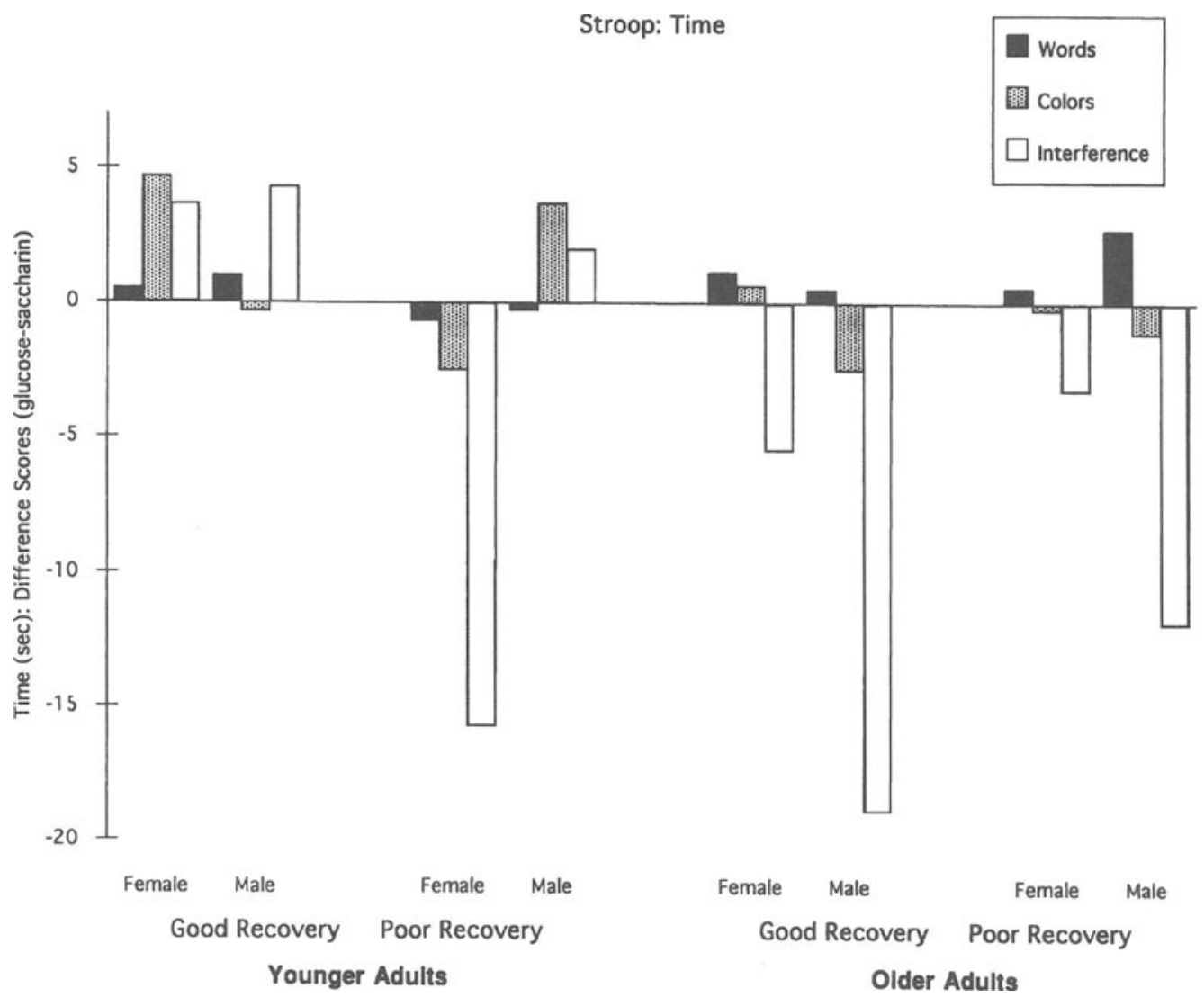

Figure 4. Mean Stroop time difference scores (glucose-saccharin) for younger and older men and women with good and poor recovery.

within-subjects factors, and age group, recovery, and sex as between-subject factors. Planned contrasts were then conducted, comparing baseline glucose levels with the four subsequent sampling times.

In general, the results showed that the older group had higher glucose levels both in the saccharin condition and after glucose administration than did the younger subjects. For all five sampling times in the saccharin condition, significant effects of age group were noted (all $p s<.01$ ), reflecting the older subjects' higher glucose levels (Figures 5 and 6). In the glucose condition, the main effects for group due to higher glucose levels in the older group were observed at 45 and 60 min following glucose administration (both $p s<.0001$ ). This pattern was further reflected in a significant interaction for glycemic condition, sampling time, and age group $[F(4,200)=5.85, p<.001]$. Planned contrasts showed that, compared with the saccharin condition, the older group had greater increases in glucose levels from baseline to $45 \mathrm{~min}$ and from baseline to $60 \mathrm{~min}$ in the glucose condition than did the younger group $[F(1,50)=10.75$ and $20.89, p \mathrm{~s}<.01$ and .0001 , respectively].

Highly significant within-subjects effects for glycemic condition and blood-sampling time were observed $[F(1,50)=726.17$, and $F(4,50)=171.10$, respectively; both $p$ s $<.0001]$. As expected, the subjects' glucose lev- els were higher after glucose administration than after saccharin administration and were higher at all sampling times compared with the baseline level in the glucose condition. In addition, significant main effects for recovery and interactions among glycemic condition, sampling time, and recovery were also observed (all $p \mathrm{~s}<$ .0001 ), as might be expected given the manner in which recovery was determined. No differences were noted between men and women in baseline glucose levels or in glucose levels following saccharin or glucose administration. No main effects for sex or interactions involving age group, recovery, and sex were observed.

\section{DISCUSSION}

As in previous studies, the effects of glucose on cognitive performance were restricted for the most part to paragraph recall, a sensitive measure of declarative memory. These effects were shown by the male subjects; the older and younger men were affected differently, according to the degree to which their glucose levels returned to baseline $60 \mathrm{~min}$ after glucose administration. No hyperglycemic effects were observed for procedural or working-memory measures or for verbal fluency. Glucose administration appeared to quicken response time and increase errors in the interference condition of the 
Glucose Levels: Saccharin Condition

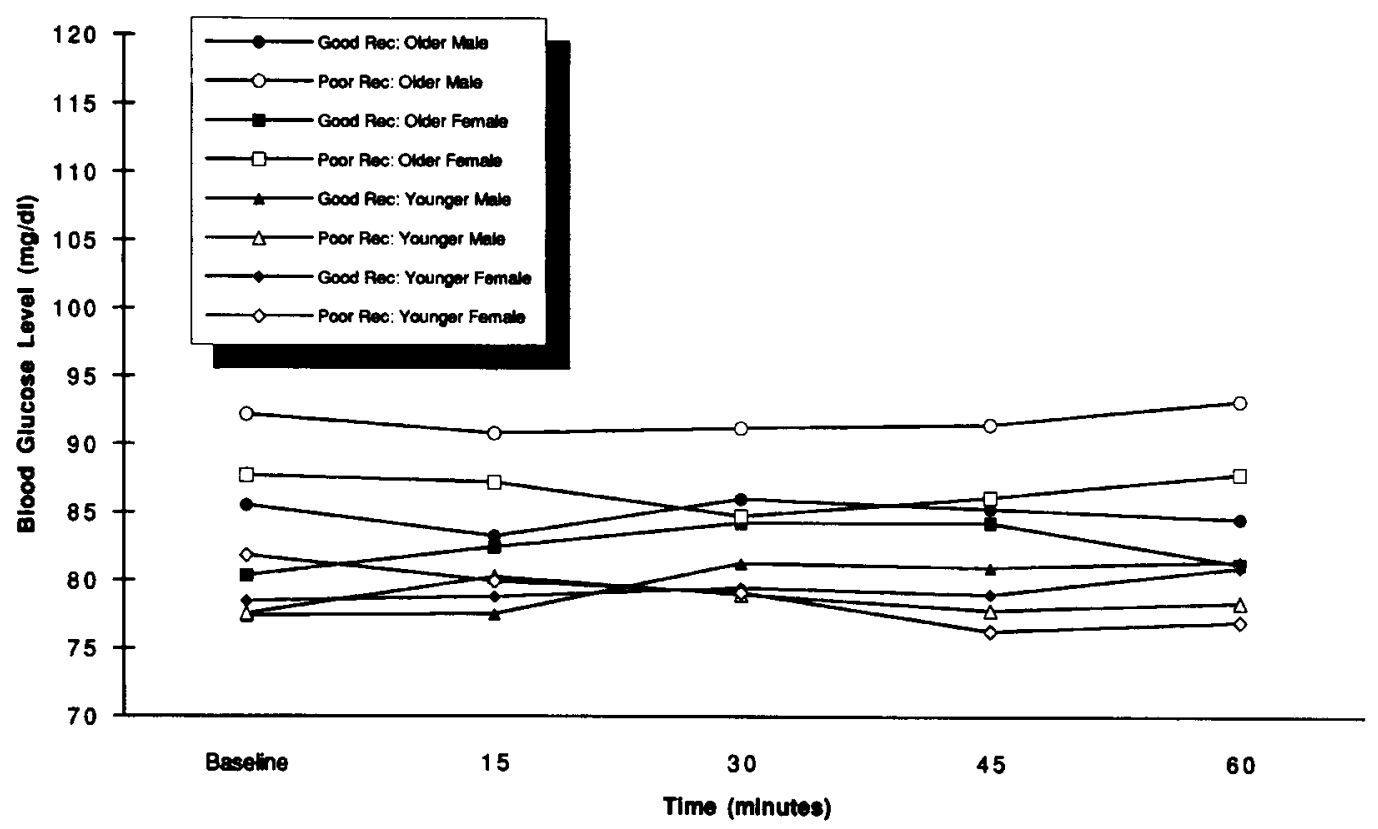

Figure 5. Mean blood glucose levels in the saccharin condition for younger and older men and women with good and poor recovery.

Glucose Levels: Glucose Condition

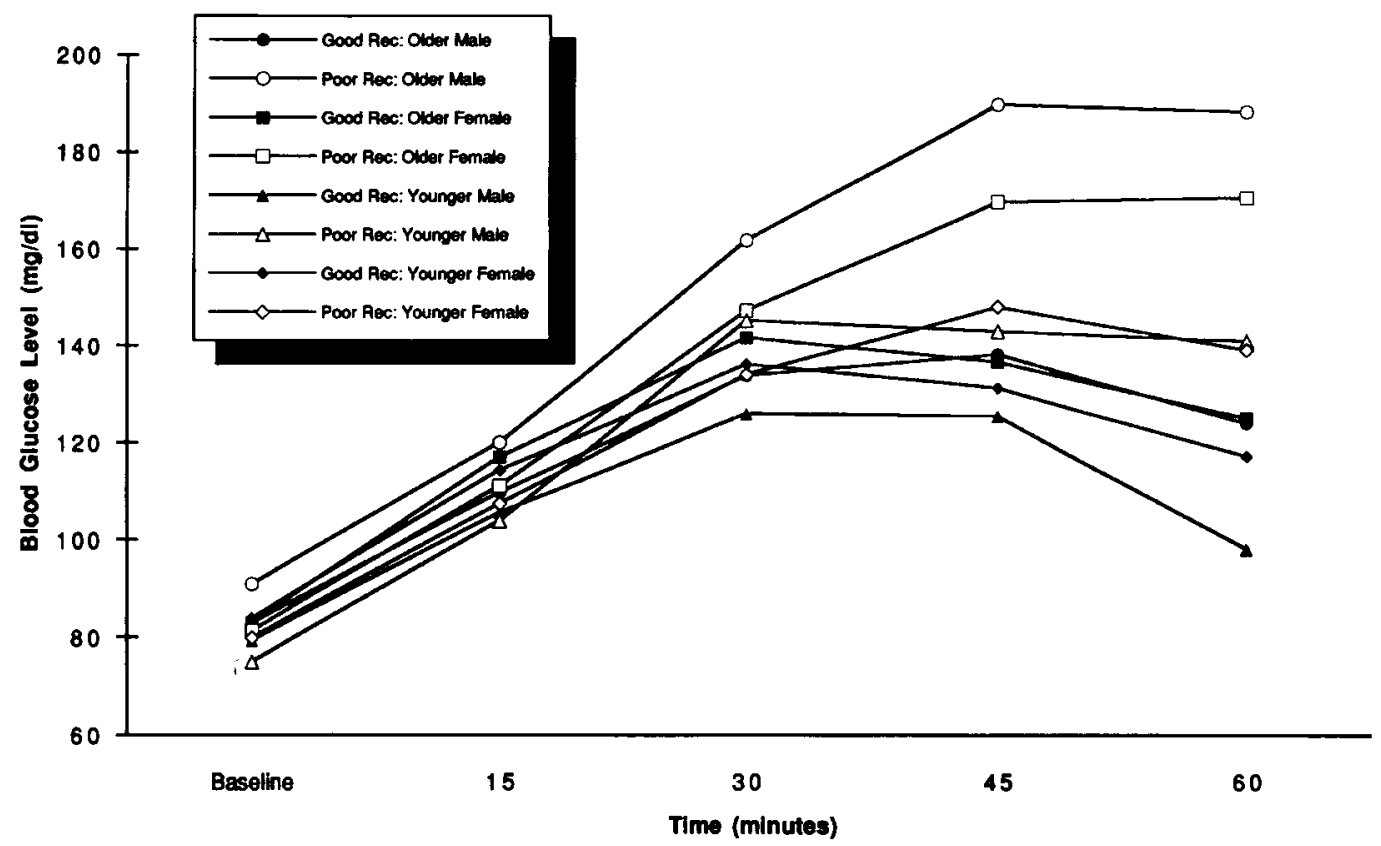

Figure 6. Mean blood glucose levels in the glucose condition for younger and older men and women with good and poor recovery . 
Stroop task for all the subject groups. Finally, expected age effects were noted across tasks in that the older adults showed less recall, slower response times, and similar verbal fluency compared with the younger adults.

\section{Hyperglycemic Effects on Paragraph Recall}

Oral glucose administration affected performance on paragraph recall, a measure of declarative memory that has been shown to be sensitive to glucose effects in previous studies (Craft et al., 1993; Craft et al., 1992; Gonder-Frederick et al., 1987; Hall et al., 1989; Manning et al., 1990). Consistent with these investigations, in the present study, the older adults with good glucose regulation showed enhanced paragraph recall following glucose administration, whereas the older adults with poor regulation showed decreased recall. Previous studies, however, did not examine the possibility that older men and women may respond differently to glucose administration. Our results suggest that men and women differ in their responsivity to hyperglycemia; older men with good glucose regulation show greater degrees of hyperglycemic facilitation than older women.

Data from the younger subjects also suggested enhanced responsivity to glucose administration for men. The pattern of responsivity, however, was reversed for the younger men, in that those subjects whose blood levels remained elevated for more prolonged periods following glucose administration showed significant hyperglycemic memory facilitation, whereas the younger men whose levels returned quickly to near baseline had decreased recall. This finding is consistent with a recent study in which memory enhancement was observed in young men whose blood glucose levels rose prior to memory testing (Benton \& Owens, 1993). In contrast, Azari (1991) observed no changes in memory performance in young men following glucose administration. This study, however, did not relate changes in memory performance from saccharin to glucose conditions to the type of glucoregulatory index used in the present investigation.

Although the basis for the selectivity of glucose effects to older subjects in previous studies has not been specified, investigators have speculated that age-related changes in glucose regulation and utilization may render older adults more susceptible to glucose effects. An alternative formulation of this argument is that efficient glucoregulatory mechanisms prevent blood glucose levels from becoming sufficiently elevated to trigger enhancement for most younger subjects. This hypothesis is supported by the data in the present study, in that the younger men who showed the highest and most prolonged elevation in blood glucose also showed glucoseinduced memory facilitation. Although these men were designated as having less recovery than the other subjects in their age group, their blood glucose levels were comparable to those of the older men designated as having good recovery, who also showed hyperglycemic memory facilitation.
A second characteristic of the present results, however, suggests that an additional factor other than blood glucose level must play a role in producing memory facilitation. The fact that the younger women with poor recovery did not show the same enhancement pattern as the younger men, despite the fact that they had similar glucose levels, indicates that sex-related aspects of glucose regulation contribute to glucose effects on memory performance. Sex differences in systemic glucose regulation and utilization have been noted for young adults in a number of studies. For example, young men had higher rates of glucose oxidation in muscle than women following ingestion of $75 \mathrm{~g}$ of glucose (Paula et al., 1990). Cultured omental adipocytes from young men also showed increased glucose transport when exposed to glucose and insulin, compared with adipocytes taken from young women (Armoni et al., 1987). In addition, it has been shown that women require higher insulin levels than men to produce a $50 \%$ fall in glucose levels (Hale et al., 1985). These results suggest that men may have higher rates of glucose utilization than women at similar plasma glucose levels. This possibility provides a basis for the finding that younger male subjects may be more sensitive to glucose effects than female subjects. It should be noted, however, that other investigators have suggested that women have higher rates of glucose metabolism than men (Yki-Jarvinen, 1984), and that estrogen facilitates insulin-promoted glucose metabolism (Renard, Bringer, \& Jaffiol, 1993). Thus, investigations of sex differences in glucose regulation and utilization have provided somewhat inconsistent results, and careful dose-response studies are needed to help document the nature and extent of such differences. In addition, the inclusion of direct measures of estrogen and insulin levels during hyperglycemia may help clarify the relationship between sex hormones and glucoregulatory mechanisms.

Although the premise that younger men designated as having less recovery reach sufficiently high blood glucose levels to trigger memory enhancement accounts for findings of hyperglycemic memory facilitation in that group, it does not address the finding that younger men whose blood glucose levels returned closest to baseline showed decreased memory following glucose administration. It might have been expected that this latter group would show no difference in performance between the two glycemic conditions. The fact that their recall deteriorated significantly suggests that some aspect of glucose regulation was affected, interfering with memory function. Although it is not possible to ascertain what glucoregulatory process may have been affected, it is interesting to note that this group had a substantially lower mean blood glucose level at the $60-\mathrm{min}$ sampling time in the glucose condition than did any other group (Figure 6).

The present findings may help clarify occasional reports of failure to find hyperglycemic facilitation in older adults. Samples in such studies may contain high numbers of female subjects or subjects with poor glu- 
cose regulation who are likely to show less hyperglycemic memory facilitation. Similarly, a failure to examine the performance of younger subjects in terms of both sex and glucoregulatory response may result in a failure to detect significant effects. Given that women show less sensitivity to glucose administration, and men show either memory facilitation or deterioration based on recovery following glucose administration, the net change in memory performance would be zero in an aggregated group containing both male and female younger subjects with good and poor recovery.

\section{Effects of Glucose on Other Cognitive Tests}

As in previous investigations, oral administration of glucose did not affect performance on a list-learning task or on the immediate-memory trials of a patternrecall task. Given that both tasks are presumed to have a substantial declarative memory component, it appears that some aspect of the tasks prevents or obscures glucose effects. Both tasks share the characteristic of repetition of stimuli over several trials. Craft et al. (1993; Craft et al., 1992) have suggested that such a procedure may create repetition priming effects that predominate in determining recall with immediate or short-delay testing. It is interesting to note that Hall et al. (1989) found hyperglycemic facilitation for a paired associate task, in which associates were repeated over trials when recall was tested after $24 \mathrm{~h}-\mathrm{a}$ time period during which repetition priming effects were likely to have dissipated. Similarly, Manning et al. (1990) found that glucose administration enhanced memory for words that had not been repeated immediately prior to recall on a selective reminding test.

An important finding from the present study is that glucose administration did not affect performance on other cognitive tasks. The fact that no differences between glycemic conditions were noted on a very difficult test of working memory - the Paced Serial Addition Test-strongly suggests that selective hyperglycemic effects on paragraph recall are not due to task difficulty. In addition, the absence of glucose effects on verbal fluency, another on-line task that has been shown to be impaired by frontal lesions, suggests that hyperglycemic memory enhancement is not due to generalized facilitation of cognitive processing. The test of implicit or procedural memory was similarly unaffected, again supporting the specificity of hyperglycemic facilitation to declarative memory. Finally, performance in the Stroop interference condition deteriorated following glucose administration, with a general increase in errors across all subject groups. Relatively faster response times in the interference condition were also noted in this study and in a recent report from another laboratory (Benton, Owens, \& Parker, in press). The fact that the same relationship among age group, recovery, and sex does not hold for the Stroop task suggests that hyperglycemic mechanisms affecting this measure differ from those affecting paragraph recall.

\section{Mechanisms of Hyperglycemic Memory Effects}

Although the present study did not incorporate measures that directly address the question of the physiological mechanisms underlying glucose effects, two conclusions can be drawn on the basis of the behavioral data. First, the fact that hyperglycemic effects were primarily restricted to a measure of declarative memory indicates that candidate mechanisms must include some basis for selectively influencing hippocampal function. Although it is possible that future studies will reveal that glucose administration affects other nonmemory tasks, to date there has been remarkable uniformity across studies, suggesting that the largest and most reliable effects are observed for sensitive measures of declarative memory such as paragraph recall.

Second, the finding of sex differences in hyperglycemic memory facilitation may also have some implications regarding the mechanisms producing such facilitation. The younger and older men who showed facilitation did so at similar glucose levels. The women showed smaller facilitation effects that did not reach statistical significance in the present study, even though their glucose levels were comparable to those of the men whose recall improved following glucose administration. It has been noted in previous studies that one aspect of glucose regulation that could account for this pattern is that women may have lower rates of insulinpromoted glucose utilization (Hale et al., 1985). It has been suggested that hyperglycemia-induced insulin elevations may increase glucose utilization in the hippocampus and in doing so enhance hippocampally mediated memory function (Craft et al., 1993). Lower rates of insulin-induced glucose utilization observed peripherally in female subjects (Hale et al., 1985) may also occur in the hippocampus, resulting in less memory facilitation for females during hyperglycemia. This possibility is speculative, but serves to illustrate one way in which sex differences in hyperglycemic memory facilitation may occur. Future studies examining neuroendocrine responses to hyperglycemia in men and women may provide further evidence for the mechanisms underlying sex differences in memory facilitation.

\section{Conclusions}

Our results suggest that hyperglycemic enhancement of memory is not due to nonspecific improvement in the ability to perform complex cognitive tasks. Glucose administration did not affect performance on difficult and sensitive tests of working memory, procedural memory, and verbal fluency. Although further studies with tests from other cognitive domains will reinforce this conclusion, the present results suggest that hyperglycemia primarily affects declarative memory ability. In addition, our findings provide some indication that at the glucose dose incorporated in the present study, men whose blood glucose reaches optimal levels are more likely to show memory facilitation, regardless of age, than are women who obtain similar blood glucose levels. Future studies 
examining sex differences in hyperglycemic responsivity may be helpful in elucidating the mechanisms underlying memory facilitation. One candidate mechanism is the rate of insulin-induced glucose utilization, which reportedly differs between men and women. Finally, our results suggest that age, sex, and glucoregulatory response all influence hyperglycemic enhancement of memory, and thus need to be considered in studies examining such effects.

\section{REFERENCES}

Armoni, M., Rafaeloff, R., Barzilai, A., Eitan, A., \& Karnieli, E. (1987). Sex differences in insulin action on glucose transport and transporters in human omental adipocytes. Journal of Clinical Endocrinology \& Metabolism, 65, 1141-1146.

AZARI, N.P. (1991). Effects of glucose on memory processes in young adults. Psychopharmacology, 105, 521-524.

BENTON, A. L. (1968). Differential behavioral effects in frontal lobe disease. Neuropsychologia, 6, 53-60.

BENTON, A. L., \& HAMSHER, K. DES. (1978). Multilingual aphasia examination (rev. ed.). Iowa City: University of Iowa.

BENTON, D. \& OWENS, D. (1993). Blood glucose and human memory. Psychopharmacology, 113, 83-88.

Benton, D., OWEns, D. S., \& Parker, P. Y. (in press). Blood glucose enhances memory and attention in young adults. Neuropsychologia.

Craft, S., Dagogo-Jack, S. E., Wiethop, B. V., MurPhy, C., Nevins, R. T., Fleischman, S., Rice, V., Newcomer, J. W., \& Cryer, P. E. (1993). The effects of hyperglycemia on memory and hormone levels in dementia of the Alzheimer type: A longitudinal study. $B e-$ havioral Neuroscience, 107, 926-940.

Craft, S., Zallen, G., \& BaKer, L. D. (1992). Glucose and memory in mild senile dementia of the Alzheimer Type. Journal of Clinical \& Experimental Neuropsychology, 14, 253-267.

Delis, D. C., Kramer, J. H., Kaplan, E., \& Ober, B. A. (1987). The California Verbal Learning Test: Research edition. New York: The Psychological Corporation.

Delis, D. C., MCKeE, R., Massman, P. J., KRamer, J. H., Kaplan, E., \& GeTtman, D. (1991). Alternate form of the California Verbal Learning Test: Development and reliability. Clinical Neuropsychologist, 5, 154-162.

Gold, P. E., \& STONE, W. S. (1988). Neuroendocrine effects on memory in aged rodents and humans. Neurobiology of Aging, 9, 709-717.

Gold, P. E., Voor, J., \& HALL, J. L. (1986). Glucose effects on memory: Behavioral and pharmacological characteristics. Behavioral \& Neural Biology, 46, 145-155.

Gonder-Frederick, L., Hall, J. L., Vogt, J., Cox, D. J., Green, J., \& GoLD, P. E. (1987). Memory enhancement in elderly humans: Effects of glucose ingestion. Physiology \& Behavior, 41, 503-504.

Gronwall, D. M. A., \& SAmpson, H. (1974). The psychological effects of concussion. Auckland: Aukland University Press/Oxford University Press.

Hale, P. J., Wright, J. V., \& Nattrass, M. (1985). Differences in insulin sensitivity between normal men and women. Metabolism, 34, 1133-1138.
Hall, J. L., Gonder-Frederick, L. A., Chewing, W. W., Silveira, J., \& GolD, P. E. (1989). Glucose enhancement of performance on memory tests in young and aged humans. Neuropsychologia, 27, 1129-1138.

Holmes, C. S. (1990). Neuropsychological sequelae of acute and chronic blood glucose disruption in adults with insulin-dependent diabetes. In C. S. Holmes (Ed.), Neuropsychological and behavioral aspects of diabetes (pp. 123-153). New York: Springer-Verlag.

Manning, C. A., Jones, C. L., Hall, J. L., \& Gold, P. E. (1990). Glucose effects on memory and other neuropsychological tests in elderly humans. Psychological Science, 1, 307-311.

Manning, C. A., Parsons, M. W., \& Gold, P. E. (1992). Anterograde and retrograde enhancement of $24-\mathrm{h}$ memory by glucose in elderly humans. Behavioral \& Neural Biology, 58, 125-130.

Nissen, M. J., Willingham, D., \& HarTman, M. (1989). Explicit and implicit remembering: When is learning preserved in amnesia? Neuropsychologia, 27, 341-352.

PalovciK, R. A., Phillips, M. I., Kappy, M. S., \& Raizada, M. K. (1984). Insulin inhibits pyramidal neurons in hippocampal slices. Brain Research, 309, 187-191.

Parsons, M. W., \& Gold, P. E. (1992). Glucose enhancement of memory in elderly humans: An inverted-U dose-response curve. Neurobiology of Aging, 13, 401-404.

Paula, F. J. A., Pimenta, W. P., SaAd, M. J. A., Paccola, G. M. G. F., Piccinato, C. E., \& Foss, M. C. (1990). Sex-related differences in peripheral glucose metabolism in normal subjects. Diabete et $\mathrm{Me}$ tabolisme, 16, 234-239.

PERret, E. (1974). The left frontal lobe of man and the suppression of habitual responses in verbal categorical behavior. Neuropsychologia, 12, 323-330.

Raichle, M. E., Herscovitch, P., Mintun, M. A., Martin, W. R., \& PowERS, W. (1984). Dynamic measurements of local blood flow and metabolism: The study of higher cortical function in humans with positron emission tomography. Annals of Neurology, 15(Suppl.), S48-S49.

RENARD, E., Bringer, J., \& JafFiol, C. (1993). Sex steroids: Effects on the carbohydrate metabolism before and after menopause. $L a$ Presse Medicale, 22, 431-435.

RYan, C. M. (in press). Effects of diabetes mellitus on neuropsychological functioning: A lifespan perspective. In I. Grant \& K. M. Adams (Eds.), Neuropsychological assessment of neuromedical disorders: Vol. 1.

SQUIRE, L. R. (1992). Memory and the hippocampus: A synthesis from findings with rats, monkeys, and humans. Psychological Review, 99, 195-231.

STROOP, J. R. (1935). Studies of interference in serial verbal reactions. Journal of Experimental Psychology, 18, 643-662.

UNGeR, J., MCNeIll, T. H., MoXley, R. T., III, White, M., Mosi, A., \& LivingsTon, J. N. (1989). Distribution of insulin receptor-like immunoreactivity in the rat forebrain. Neuroscience, 31, 143-157.

YKI-JARVINEN, H. (1984). Sex and insulin sensitivity. Metabolism, 33, 1011-1015.

(Manuscript received September 22, 1993; revision accepted for publication December 22, 1993.) 